\title{
ALGORITMOS DE GESTIÓN DE PERSONAL ENFOCADOS A LA MEJORA DEL SERVICIO AL CLIENTE. APLICACIÓN A SERVICIOS DE URGENCIAS DE ATENCIÓN PRIMARIA
}

\section{ALGORITHMS OF STAFF MANAGEMENT FOCUSED TO THE IMPROVEMENT OF THE CUSTOMER SERVICE. APPLICATION TO PRIMARY ATTENTION EMERGENCY SERVICES}

\section{ABSTRACT:}

This paper presents a methodology for planning and organizing resources in the context of a medical clinic emergency. This methodology can be applied to other type of services and is implemented in software that effectively solves the planning of certain tasks. Planning the primary attention emergency services (to improve their quality and to be attractive for patients) can clear the hospital emergency rooms, influencing in an integral way the whole sanitary emergency service. The hospital emergency services receive a considerable proportion of patients who should be assisted in the emergency services for primary attention. A reduced wait time is the main factor that patients identify with quality of service. Therefore, optimizing this element is an option to reach this aim. This has led to the development of an algorithm which aims to not exceed a prearranged wait time in the emergency services of primary attention, and gets as a response the distribution of personnel to be assigned to optimize the consumption of resources. Additionally, it is given a second computational development which transforms the previous theoretical assignment of personnel into another one that is easily applicable in practice by the agents of the services. It has developed a plan that calculates the number of doctors needed in an emergency service of primary attention, once the maximum wait time desired of this service has been determined. The plan is a tool for the management and planning of the number of doctors and their activity in an emergency service of primary attention, depending on the desired wait time, which adapts to the dynamic and changing features which these services present due to their application in a wide variety of production sectors. Keywords:public services management, resource optimization,organizational structure, algorithms

\section{RESUMEN:}

En el presente artículo se desarrolla una metodología de planificación y organización de recursos en el contexto de las urgencias de un centro de salud que puede ser extrapolada a otro tipo de servicios Dicha metodología desemboca en la realización de un programa de ordenador que resuelve de forma efectiva ciertas tareas de planificación. Planificar los servicios de urgencias de atención primaria (SUAP) para que mejoren su calidad y sean atrayentes para los pacientes puede descongestionar las urgencias hospitalarias incidiendo de manera integral en todo el servicio de urgencias sanitario. El tiempo de espera reducido es el principal factor que los usuarios identifican con la calidad del servicio, por ello actuar sobre este elemento supone una opción para lograr este fin. Se presenta el desarrollo de un algoritmo que, teniendo como objetivo no sobrepasar un tiempo de espera prefijado en un SUAP, obtenga como respuesta la distribución de personal a asignar optimizando además el consumo de recursos. Adicionalmente, se aporta un segundo desarrollo computacional que transforma la asignación teórica de personal anterior en otra que sea fácilmente aplicable en la práctica por los gestores de los servicios. De esta forma, se ha desarrollado un programa que calcula el número de facultativos necesarios en un SUAP, una vez que se ha fijado el tiempo máximo de espera deseable en dicho servicio. El programa constituye un instrumento de gestión y planificación del número de médicos y su actividad en un SUAP, dependiendo de los tiempos de espera deseados, que se adapta a las características dinámicas y cambiantes que dichos servicios presentan por su naturaleza, siendo de aplicación en un amplio abanico de sectores productivos.

Palabras clave: gestión de servicios públicos, optimización de recursos, organización de empresas, algoritmos 


\section{1.- INTRODUCCION}

Desde hace décadas es incuestionable la similitud existente entre un centro sanitario y un establecimiento industrial. El sector sanitario se debe considerar como un sector industrial y deben fluir entre ambos de manera bidireccional técnicas económicas de análisis y desarrollo[1].

Hoy en día, la sanidad ocupa una de las posiciones más relevantes en cuanto a recursos consumidos y personal asociado al sector,además de ser uno de los principales asuntos de preocupación de la sociedad. Analizar el sistema sanitario como una empresa que ofrece un servicio a clientes, y que busca la mejora del servicio con la optimización de los recursos humanos consumidos, supone por un lado realizar un análisis empresarial y por otro, que el estudio realizado pueda extrapolarse fácilmente a otro tipo de sectores.

Particularmente, en este trabajo, se analiza la gestión de los servicios de urgencia de atención primaria como una unidad que interactúa con los servicios de urgencia hospitalarios.

La masificación de los servicios de urgencias hospitalarios (SUH) es una realidad creciente, que refleja los problemas de adecuación en la utilización de dichos servicios y las deficiencias en la coordinación entre éstos y los servicios de atención primaria de salud $[2,3,4]$.

Los SUH realizan tareas propias de la atención primaria y de otros niveles no urgentes de atención[2,3], lo cual genera una serie de disfunciones que afectan a la organización y la eficiencia de los hospitales, a los usuarios y al conjunto del sistema sanitario [4].

La actitud del paciente de acudir como primera opción a un servicio de urgencias hospitalario no se debe tomar sólo como una actitud aprendida o como una muestra de los cambios socio-laborales de la sociedad, sino como una crítica al sistema sanitario actual que no da las respuestas que la sociedad demanda. No sólo hemos de considerar la educación sanitaria dirigida a un adecuado uso de los servicios sanitarios como la herramienta a usar para paliar el uso de las urgencias hospitalarias como primera opción del usuario, se ha de considerar la necesidad de evaluar al propio sistema sanitario para dar una respuesta más eficaz al ciudadano [5].

La masificación de las urgencias médicas hospitalarias provoca problemas económicos y de calidad asistencial:

- el coste de una misma enfermedad no urgente atendida en un SUH es entre 2.5 y 3 veces superior que si se atiende en una consulta no urgente [6].

- aumentan los tiempos de espera lo que disminuye la eficiencia del servicio y la calidad asistencial.

Una solución a este problema es el estudio del servicio de urgencias médicas de manera integral, teniendo como principal objetivo que un porcentaje de los pacientes que ahora mismo acuden al SUH como primera opción acudan al SUAP, lo cual descongestionaría el servicio hospitalario y reduciría los costes globales de los servicios de urgencias.

Sin embargo, para que la población revierta su conducta de acudir en primera opción a los centros hospitalarios se les debe ofrecer un servicio de calidad en los centros de salud.

Según $[7,8,9]$ la idea de calidad de servicio viene determinada principalmente por un tiempo de espera reducido. Las esperas prolongadas constituyen una de las principales causas de insatisfacción de los usuarios de estos servicios siendo fuente frecuente de reclamaciones. Por ello los tiempos de espera constituyen un importante método de control de calidad para las empresas que realizan servicios con atención al público.

Si se consiguen gestionar adecuadamente las urgencias de los centros de atención primaria para que se comprometan a no sobrepasar tiempos de espera razonables, se estaría dando un gran paso en posicionar esta atención por delante de la hospitalaria para patologías no graves.

En la resolución de este problema, la gestión de tiempos de espera de manera integrada con la optimización de recursos de personal médico es de vital importancia, ya que se trata de servicios críticos en los que debe intentarse, por un lado 
que los tiempos de acceso a la atención de los pacientes estén dentro de unos márgenes razonables, y por otro que el número de personal involucradopara la consecución de ese tiempo de espera sea óptimo, equilibrando así una atención de calidad con un coste en recursos sostenible. Por ello, la organización y planificación de estos servicios debe ser un tema de interés en Sanidady otros sectores $[10,11]$.

En el presente artículo se desarrolla una metodología de planificación y organización de recursos en el contexto de las urgencias de un centro de salud que puede ser extrapolada a otro tipo de servicios. Dicha metodología desemboca en la realización de un programa de ordenador que resuelve de forma efectiva ciertas tareas de planificación. En concreto, el problema de optimización a resolver es encontrar el número mínimo de médicos que necesita un centro de salud para cubrir el servicio de urgencias, consiguiendo que el tiempo de espera requerido sea el adecuado para el buen funcionamiento del servicio. Este estudio además permite analizar las franjas horarias de atención médica que son responsables potenciales de las demoras si no se aumenta la cantidad de personal en ellas.

\section{2.- MATERIALES Y METODOS}

El modelo que se presenta se centrará en la organización del SUAP, pudiendo adaptarse al periodo deseado: días completos (urgencias de sábados y festivos) o periodos inferiores (nocturnos en días laborables).

El objetivo principal consiste en conseguir atender a todos los pacientes que acudan al servicio de urgencias sin que ninguno de ellos sobrepase el tiempo de espera máximo que se proponga en la planificación, todo ello utilizando el menor número posible de recursos, es decir, se quiere minimizar el uso de horas de médico manteniendo el compromiso de no sobrepasar el máximo tiempo de espera prefijado. Además se organizará su horario de trabajo para poder hacer frente a momentos críticos en la afluencia de pacientes, así como a las necesidades de descanso de los facultativos.

Para ello, un paciente debe ser reconocido en el menor tiempo posible, es decir, por el primer médico que quede libre, pero no podrá recibir atención hasta que se haya hecho lo mismo con los pacientes anteriores. La clasificación de pacientes según su gravedad, también llamada "triaje" no está implantada en los SUAP, principalmente debido a la mayor incidencia de enfermedades de una patología menor, por lo que no serían extrapolables los modelos clásicos de "triaje" utilizados en otros servicios de emergencias, por ello en este estudio todos los pacientes son considerados homogéneos y el tiempo medio de atención sanitaria por paciente definido abajo es una medida que les representa a todos.

El momento en el cual un paciente puede comenzar a recibir atención médica, determina su tiempo de espera en el servicio (tw) y depende de factores que han sido fijados previamente por las autoridades y factores sobrevenidos. De esta forma podemos decir entonces que el tiempo de espera sufrido por cada paciente es debido a:

- Factores fijados previamente por las autoridades:

- tiempo medio de atención sanitaria por paciente $\left(\mathrm{t}_{\mathrm{m}}\right)$ : las autoridades suponen que un médico dispone de $\mathrm{t}_{\mathrm{m}}$ minutos para atender a un paciente.

- número de médicos existentes en el servicio sanitario (n): las autoridades contratan un número determinado de médicos para atender el servicio.

- Factores sobrevenidos:

- número de pacientes que acuden al servicio de urgencias en los diferentes tiempos.

El estudio que aquí se presenta tiene como objetivo lograr no sobrepasar el tiempo de espera máximo exigido al servicio. Los datos de entrada son el tiempo de espera máximo, tiempo medio de atención sanitaria por paciente y datos estadísticos de afluencia de pacientes a servicios de urgencia, teniendo como resultado el número de médicos exigibles y sus horas de utilización.

El modelo se desarrolla calculando el instante en el que cada paciente es atendido por el médico, computando así su tiempo de espera total en el servicio médico.

Se considera la hipótesis de que en determinados instantes de tiempo $t 1_{\mathrm{i}}$ se actualizan los datos de los nuevos pacientes que han llegado al servicio de urgencias. En cada uno de estos instantes se registra la entrada de $\mathbf{J}_{\mathbf{i}}$ pacientes que pasan a 
la última posición de la lista de espera. Los pacientes serán atendidos en el orden en el que se encuentran en la lista por uno de los $n$ médicos que prestan servicio en el centro.

La nomenclatura utilizada en el modelo es la siguiente:

- $\quad t l_{\mathrm{i}}$ : momento en el que entran en lista de espera $\mathrm{J}_{\mathrm{i}}$ pacientes.

- $\quad \mathrm{P}_{\mathrm{k}}^{\mathrm{i}}$ : paciente $\mathrm{k}$-ésimo $\mathrm{k} \in\left\{1, \ldots, \mathrm{J}_{\mathrm{i}}\right\}$ del total de $\mathrm{J}_{\mathrm{i}}$ pacientes que entran en el servicio de urgencias en el instante $\mathrm{tl}_{\mathrm{i}}$.

- $\quad t 2_{\mathrm{ik}}$ : momento en el que es atendido el paciente k-ésimo del conjunto de pacientes que entran en el instante $\mathrm{t} \mathrm{l}_{\mathrm{i}}$

- $\quad$ tw_ $\mathrm{P}_{\mathrm{k}}^{\mathrm{i}}=\mathrm{t} 2_{\mathrm{ik}}-\mathrm{t} 1_{\mathrm{i}}:$ tiempo de espera del paciente $\mathrm{P}_{\mathrm{k}}^{\mathrm{i}}$.

Como aplicación del modelo, se trata el caso general de un centro de atención primaria que, en su horario de urgencias, dispone de $n$ facultativos. Se dan las siguientes opciones de estudio:

a. En el momento de contabilización de los pacientes en el servicio de urgencias $\left(\mathrm{t}_{\mathrm{i}}\right)$, los médicos están ocupados con pacientes anteriores. El instante en el que un paciente $\mathrm{k}$, comienza su atención médica $\left(\mathrm{t} 2_{\mathrm{ik}}\right)$ se retrasa en función de los momentos de atención de los pacientes que le preceden. El paciente comienza su atención en consulta en el mínimo de los tiempos de salida de losn pacientes inmediatamente anteriores a él. Este hechoqueda representado en la Fig.1(a).En ella, cada barra (rectángulo coloreado que representa el tiempo efectivo de atención en consulta) se desplaza horizontalmente hasta posicionarse a continuación de la última barra (de entre todas las consultas) que esté situada más a la izquierda.

b. En el momento de contabilización de los pacientes en el servicio, existen $m$ médicos libres $1 \leq m \leq n ; m$ pacientes se asignan a estos facultativos posicionando su barra de atención a partir de ese instante, el tiempo de espera es tw $\mathrm{P}_{\mathrm{k}}^{\mathrm{i}}=\mathrm{t} 2_{\mathrm{ik}}-\mathrm{t} 1_{\mathrm{i}}=0, \mathrm{k} \in\{1, \ldots, m\}$. Si $\mathrm{J}_{\mathrm{i}}>m$ existen $\mathrm{J}_{\mathrm{i}}-m$ pacientes que serán atendidos no inmediatamente, según lo indicado en a. Obsérvesela zona marcada con barra verde que representa el tiempo que el médico está libre. Estos hechos quedan representados en la Fig.1(b).
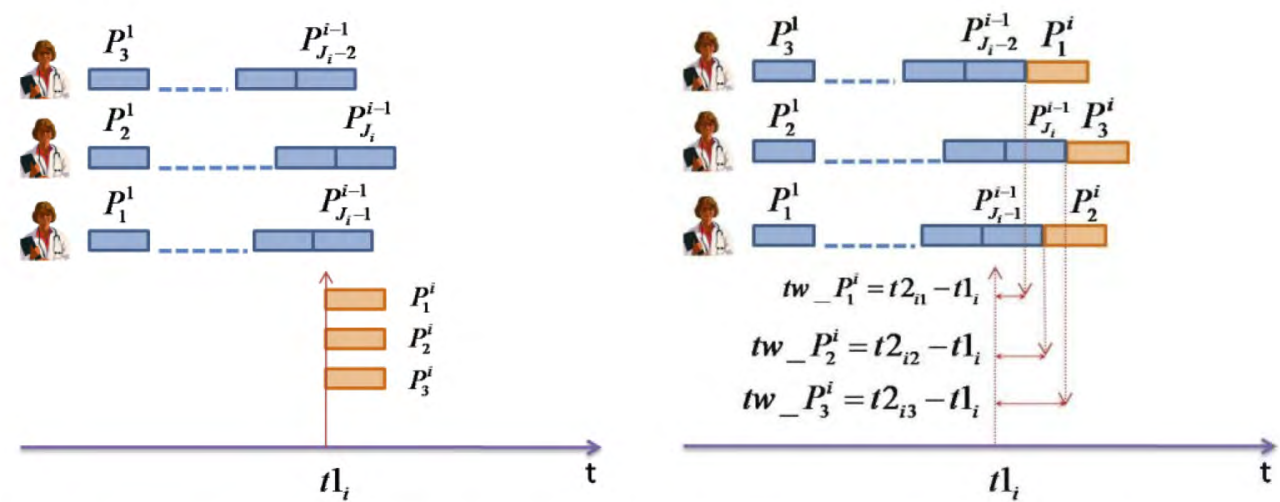

Fig. I(a).Entrada y posicionamiento de pacientes en el instante $t l_{i}$ para $n$ médicos ocupados
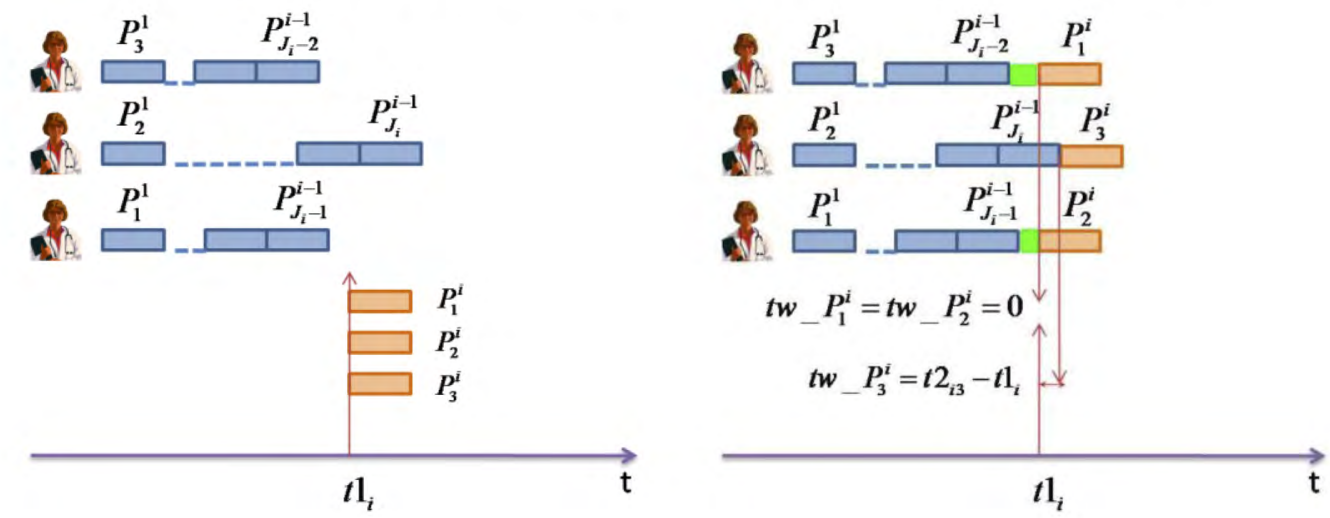
Fig. l(b). Entrada y posicionamiento de pacientes en el instante $t_{i}$ para n médicos existiendo algún médico libre.

\section{3.- RESULTADOS}

Se ha desarrollado un programa que, a partir de la metodología expuesta en la sección anterior, calcula el número de facultativos necesarios en el servicio, una vez que se ha fijado el tiempo máximo de espera deseable en dicho servicio (tiempo máximo permitido para el mantenimiento de los estándares de calidad). De esta forma, las variables de entrada y salida para dicho programa serán:

Input:

- Tiempo máximo de espera deseable en el servicio.

- Tiempo medio de atención a los pacientes por el facultativo $\left(\mathrm{t}_{\mathrm{m}}\right)$.Este tiempo medio es una variable que permite adaptarse a las necesidades de cada centro según su distribución de tiempo de atención a sus pacientes. Usualmente se utilizan distribuciones como la lognormal para modelar los tiempos de atención en el área de salud[12].

- Datos de afluencia de pacientes: número de pacientes que entran en el servicio de urgencia en cada instante en el que se actualiza la lista de entrada.Los datos suministrados en este input permiten obtener de forma implícita los tiempos en los que se actualiza la lista de espera. Ambas variables están contenidas en esta entrada lo que permite la adaptación de ambas a las necesidades de cada centro.

Output:

- Mínimo número de médicos simultáneos en el servicio para que pueda garantizarse el tiempo de espera máximo deseado

\section{Generación de los datos de afluencia de pacientes:}

Según datos suministrados por el Ministerio de Sanidad (año 2011), el sistema público atendió hasta 27 millones de urgencias en los centros de atención primaria. Esto supone que para un centro de salud asociado a una población de 200.000 habitantes, el número de urgencias atendidas en un intervalo de 24 horas se puede estimar en 500 personas.

Se conoce además que el 80\% de la afluencia de pacientes se produce entre las 10:00 y las 24:00 horas, presentándose dos picos entre las 10:00 y las 12:00, y entre las 16:00 y las 18:00 horas[13]

Con estos datos de entrada, se ha generado la distribución de afluencia de pacientes en franjas de 10 minutos, para un periodo de 24 horas de servicio de urgencias de atención primaria. No obstante, al ser la unidad de tiempo en que se actualiza la lista de entrada una variable del programa, cada centro de salud puede elegir la franja de tiempo en que contabiliza la entrada de pacientes según sus necesidades, para lograr una mayor efectividad en el servicio prestado.

Para generar dicha afluencia de pacientes se ha supuesto, como es habitual en estos modelos [14],que ésta sigue una distribución de Poisson de parámetro el mismo de una distribución uniforme fijada por la afluencia de pacientes en un día completo para las franjas de tiempo prefijadas. Para la simulación de datos se aplica el método de simulación de Montecarlo que permite la generación de variables aleatorias [15] - utilizando 100 repeticiones.

La afluencia de pacientes generada queda representada gráficamente en la Fig.2. 


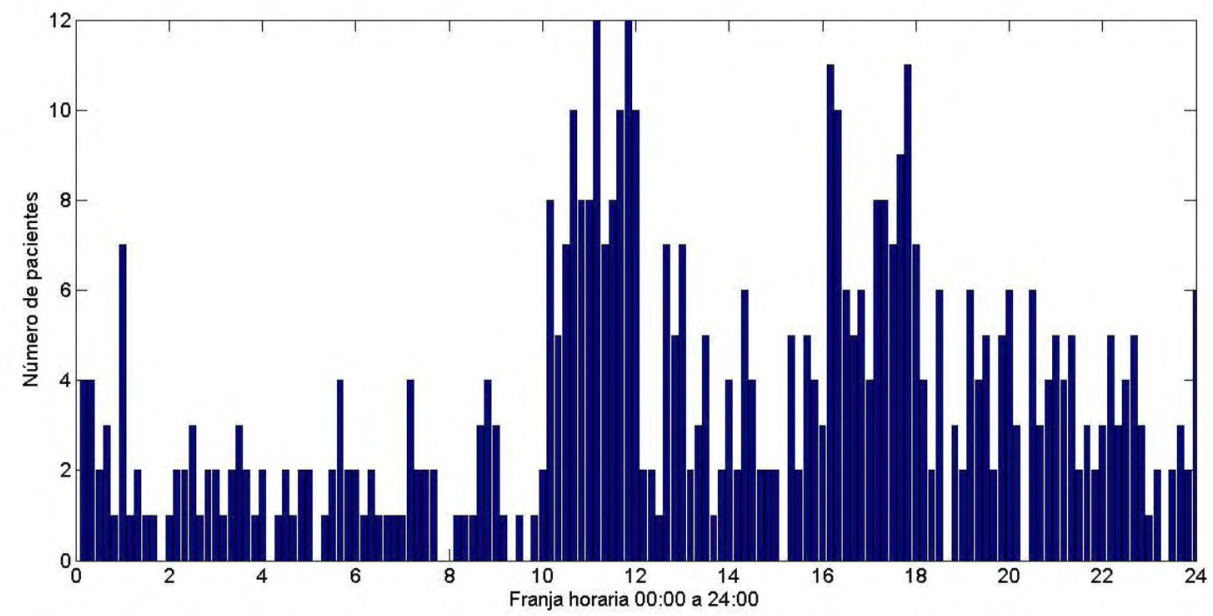

Fig.2. Afluencia de pacientes por intervalos de 10 minutos en un periodo total de 24 horas

El programa se ha implementado utilizando MATLAB 2014b. A continuación se escribe el pseudocódigo utilizado:

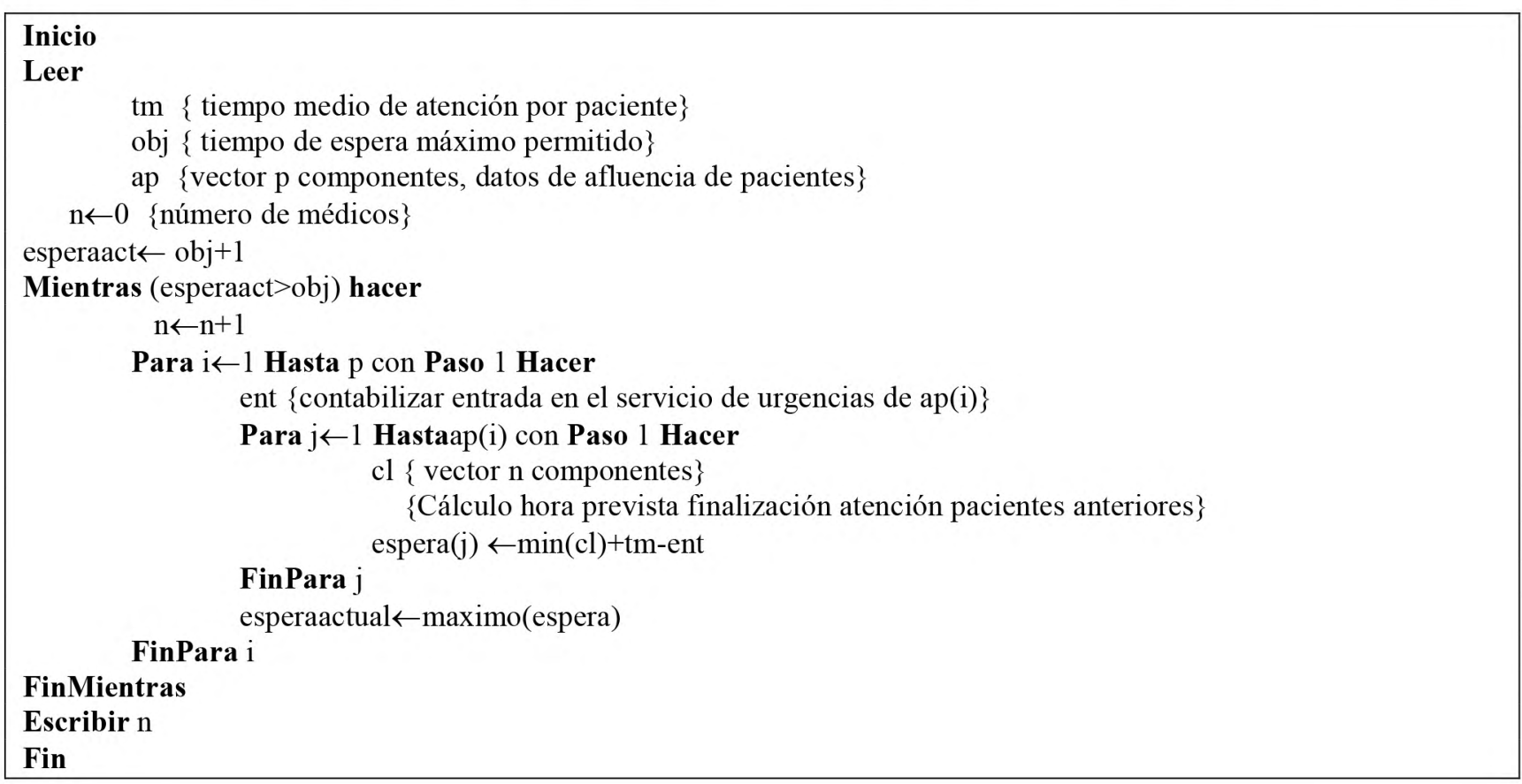




\section{Desarrollo computacional: paso 1}

Se presenta a continuación una implementación en la que se ha considerado un tiempo medio de atención, tm, de 10 minutos. El tiempo de espera máximo que se propone como objetivo es de 40 minutos. Estos datos pueden modificarse adaptándose a las necesidades de cada centro.

El programa da como respuesta que el logro del objetivo se consigue con 7 médicos simultáneos siendo la media de médicos utilizados en cada instante a lo largo de las 24 horas de 3.47. Los resultados detallados se ofrecen en forma gráfica en Fig.3 y Fig.4.

En la Fig.3(a) se observa una franja horizontal para cada uno de los siete médicos, marcando en color azul el tiempo efectivo en el que está en consulta y en verde el tiempo en el que no está atendiendo pacientes. Se aprecia que el tiempo en que están ocupados pasa por dos zonas de hora punta, una en la mañana y otra en la tarde, tal como era de esperar por la afluencia de pacientes. En el resto de horas no es óptimo económicamente, ni necesario, la presencia del número máximo de facultativos. Esta circunstancia se vuelve a mostrar en la Fig.3(b), en la que aparece el número de médicos simultáneos usados en cada instante. Se observa que excepto para las zonas horarias de mayor afluencia, se puede lograr el objetivo con menos facultativos.

En la tabla I se puede observar el tiempo en minutos en el que se está usando simultáneamente de 0 a 7 médicos. A lo largo del día sólo se utilizan 7 médicos simultáneos durante 320 minutos, siendo la media de facultativos utilizados en el resto del día de 2.46 .

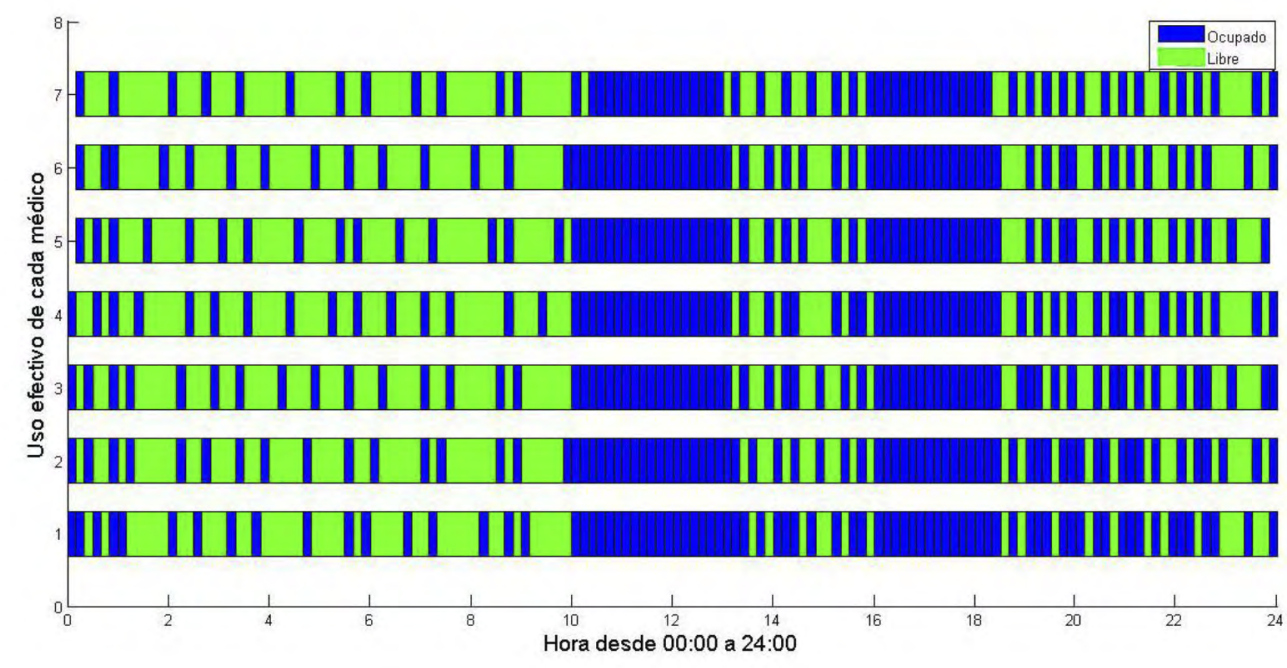

Fig. 3(a). Ocupación de cada uno de los médicos. 


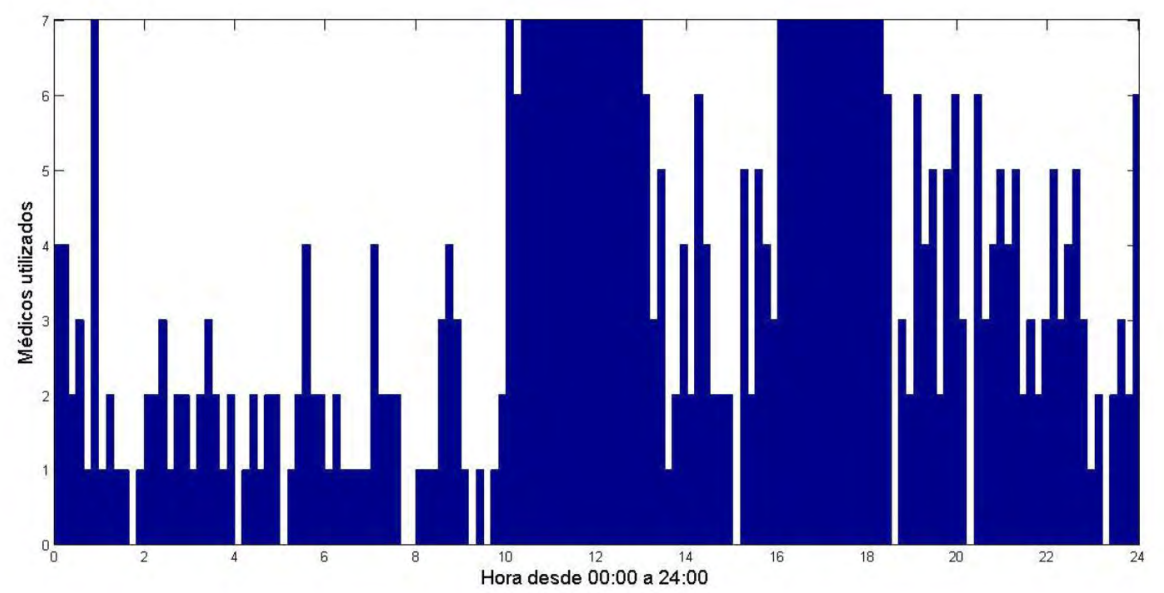

Fig. 3(b).Número de médicos utilizados en cada instante

\begin{tabular}{|c|c|}
\hline Número de médicos simultáneos & Minutos utilizados \\
\hline 0 & 110 \\
\hline 1 & 240 \\
\hline 2 & 330 \\
\hline 3 & 150 \\
\hline 4 & 120 \\
\hline 5 & 90 \\
\hline 6 & 80 \\
\hline 7 & 320 \\
\hline
\end{tabular}

Tabla I. Minutos en que se utilizan simultáneamentecada grupo de médicos

A continuación, en la Fig.4, se muestra el tiempo de espera que sufren los pacientes en cada instante, a lo largo de las 24 horas de servicio. Salvo en las horas punta en las que se llega a alcanzar el tiempo máximo de espera permitido, en las horas restantes se trabaja sin espera, lo cual nos indica que se puede reducir el número de médicos que se están utilizando en esas franjas horarias y que se indican en la Fig.3. 


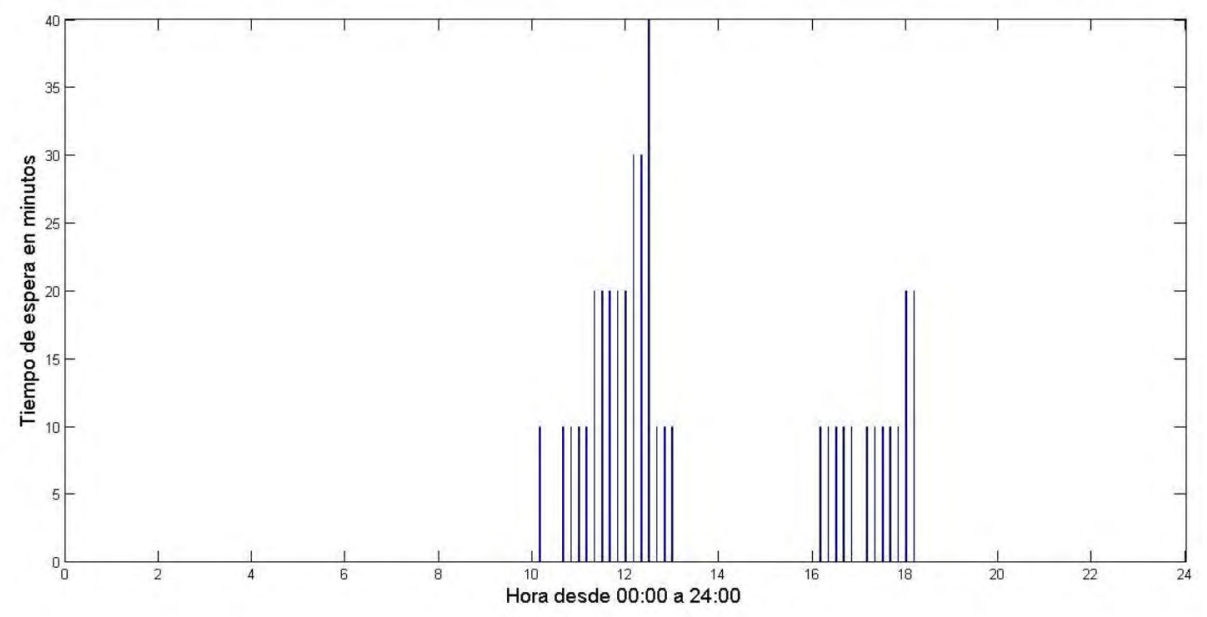

Fig.4. Tiempo de espera real en cada instante

\section{Desarrollo computacional: paso 2}

A la hora de planificar un servicio de urgencias no es posible manejar un cambio continuo en el número de médicos asignado al servicio como el que da como resultado el primer paso del experimento y que se ofrece en la Fig.3. Se debe por tanto llegar a una solución que sea eficiente en cuanto a la utilización de recursos y a la puesta en práctica de éstos.

Teniendo como datos de partida los resultados del primer desarrollo computacional, es decir, manejar un número máximo de siete consultas abiertas simultáneas y que la media de facultativos empleado a lo largo de las 24 horas de servicio es de 3.47, se ha desarrollado un segundo algoritmo en el que se trata de llegar a una distribución de facultativos más estable a lo largo del tiempo. De esta forma, las variables de entrada y salida para dicho programa serán:

Input:

- Afluencia de pacientes

- Tiempo medio de atención por paciente

- Tiempo de espera máximo permitido

- Distribución inicial de médicos según Fig.3

Output:

- Distribución final de médicos

- Media real de médicos utilizada

- Pacientes que superan la espera máxima

Este programa, al igual que el anterior, se ha implementado con MATLAB 2014b.El pseudocódigo utilizado se indica a continuación:

\section{Inicio}

Leer

tm \{ tiempo medio de atención por paciente\}

obj \{tiempo de espera máximo permitido\}

ap $\{$ vector $\mathrm{p}$ componentes, datos de afluencia de pacientes\}

nmed \{vector $\mathrm{p}$ componentes, número de médicos obtenido paso 1 \} 


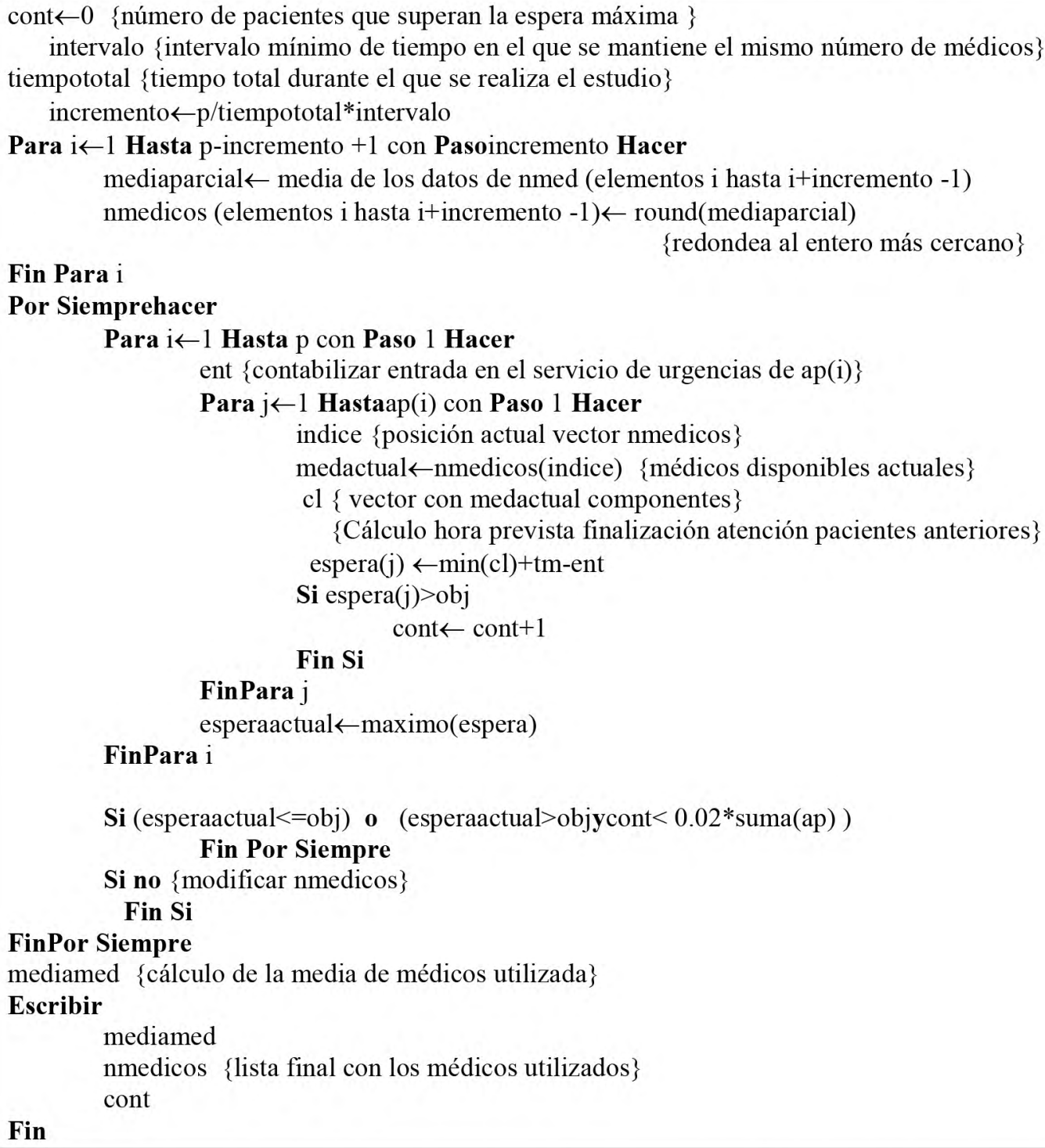

Se trata de encontrar una distribución de médicos manejable por la gerencia del sistema con una media cercana a la obtenida y que no sobrepase el tiempo máximo de espera. Dada la aleatoriedad del proceso se acepta que el tiempo de espera sobrepase mínimamente el tiempo objetivo (40 minutos) sólo para un número reducido de pacientes (se estima en el $2 \%$ del total de pacientes atendidos en el periodo completo).

Con estas condiciones, la puesta en práctica del algoritmo arroja los siguientes resultados: media de médicos utilizados 3.84, tiempo de espera máximo 40 minutos (salvo para 5 pacientes a lo largo del día en los que se llega a 50 minutos). La distribución final de médicos es la dada en la Fig.5.

En la tabla II se observa que el número de consultas abiertas se mantiene estable durante periodos largos de tiempo. 


\begin{tabular}{|c|c|}
\hline Número de consultas abiertas & Periodo \\
\hline 2 & $00: 00-10: 00$ \\
2 & $22: 10-24: 00$ \\
\hline 4 & $18: 00-22: 10$ \\
\hline 6 & $12: 00-16: 00$ \\
\hline 7 & $10: 00-12: 00$ \\
\hline 7 & $16: 00-18: 00$ \\
\hline
\end{tabular}

Tabla II. Detalle horario de la distribución final de médicos

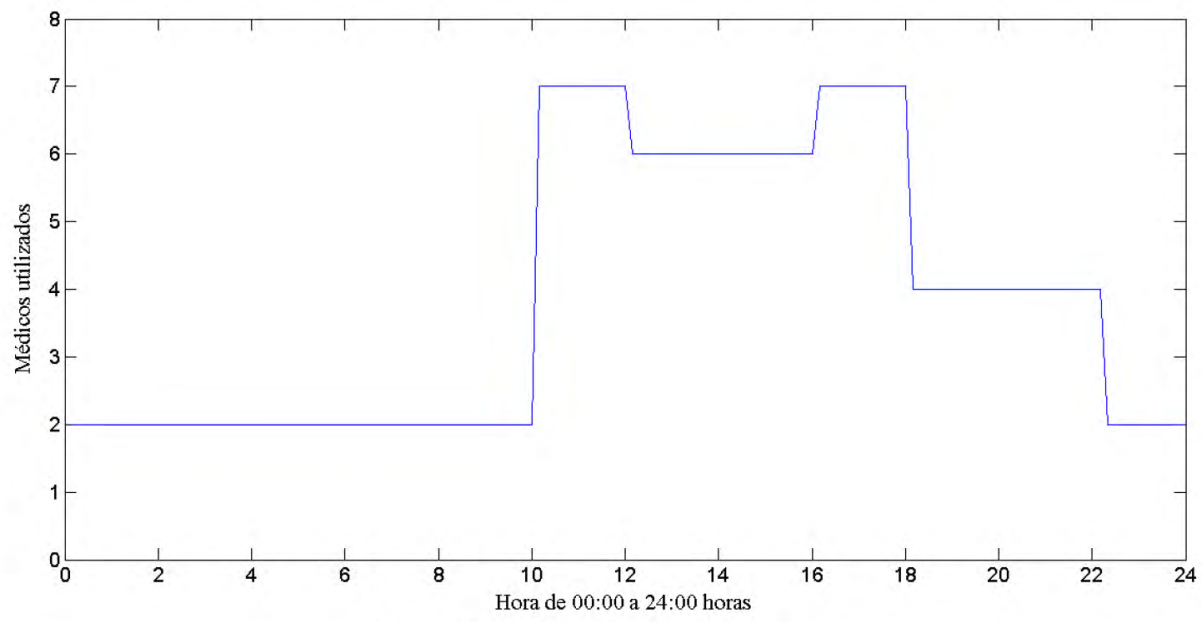

Fig.5. Distribución final de médicos

\section{4.- DISCUSION}

En el presente trabajo se ha desarrollado e implementado un software que consigue una planificación de los tiempos de espera de un SUAP adecuando y optimizando los recursos. Se obtiene como resultado la distribución horaria de personal médico a lo largo del periodo estudiado lo que supone una herramienta fundamental para la gerencia de estos servicios que pueden planificar los horarios de trabajo de los facultativos con el objetivo del mantenimiento de los estándares de calidad, siendo de aplicación en un amplio abanico de sectores productivos.

El algoritmo que se presenta, como la mayoría de los programas de ordenador, se ajusta a los principios básicos de planificación[16]. Las variables que se definen en él cuyo rango puede ser elegido en cada momento por el usuario, permiten que se adapte a diferentes situaciones dando al procedimiento un carácter dinámico y flexible. Constituye un instrumento de gestión y planificación del número de médicos y su actividad en un SUAP, dependiendo de los tiempos de espera deseados, que se adapta a las características dinámicas y cambiantes que dichos servicios presentan por su naturaleza. Supone así una alternativa a otros métodos de estudio de este tipo de problemas como son las líneas de espera (teoría de colas) y simulación de eventos discretos[17]. 
Dado que para la misma dolencia, el coste de atención en los SUH es mayor que en los SUAP, desarrollar herramientas como la presentada que mejoren dicho servicio e invertir en el aumento de personal en los SUAP puede producir rentabilidad económica en la globalidad del servicio.

Este trabajo puede abrir una línea futura de actuación, ampliando el estudio con la optimización de otros factores que pueden influir en el desarrollo de un SUAP y redundar en la mejora del servicio del mismo, como pueden ser la optimización del personal de apoyo o el personal administrativo entre otros.

Por otro lado, puede ampliarse el horizonte del estudio presentado aplicándose a la optimización de otros tipos de recursos hospitalarios como puede ser, entre otros, el servicio de ambulancias [18].

\section{BIBLIOGRAFÍA}

[1] Peña Sánchez de Rivera. "Ingeniería y medicina". I Congreso español de ingeniería industrial. Valencia 1977. Premio DYNA.

[2] Gómez-Jiménez J, Becerra O, Boneu F, et al. "Análisis de la casuística de los pacientes derivables desde urgencias a atención primaria". Gaceta Sanitaria.2006. Vol 20-1.p.40-46.DOI: http://dx.doi.org/10.1157/13084126.

[3] Jiménez L, Hermoso F, Tomás S, et al. "Urgencias sanitarias en España: situación actual y propuestas de mejora". Sociedad Española de Medicina de Urgencias y Emergencias. Granada: Escuela Andaluza de Salud Pública. 2003.

[4] Peiró S, Sempere T, Oterino D. "Efectividad de las intervenciones para reducir la utilización inapropiada de los servicios hospitalarios de urgencias. Revisando la literatura 10 años después del informe del defensor del pueblo". Economía y Salud. 1999. Vol.33. p.3-12.

[5] Núñez R, Leo B, Aragón D, Carballo JM, et al. "Por qué acuden los pacientes a urgencias hospitalarias". Revista científica de la sociedad española de enfermería de urgencias y emergencias. 2009. Vol.8 p.8. http://www.enfermeriadeurgencias.com/ciber/julio2009/pagina8.html

[6] Cunningham PJ, Clancy CM, Cohen JW, et al."The use of hospital emergency departments for nonurgent health problems: a national perspective". Medical CareResearch and Review. 1995. Vol.52-4.p.453-74.DOl:http://dx.doi.org/10.1177/107755879505200402.

[7] Miró O, Sánchez M, Coll-Vinent B, et al. "Indicadores de calidad en Urgencias: comportamiento en relación con la presión asistencial". Medicina Clínica. 2001. Vol. 116-3. p.92-97.

[8] Rius C, Vaquero F, Saguer M. "Estudio del tiempo de estancia en el Servicio de Urgencias como sistema de control de calidad". Emergencias. 1990. Vol.2-1. p.22-25.

[9] Surroca RM, Simón RM, Juix J, et al. "Metodología de análisis de calidad de un Servicio de Urgencias Hospitalario". Revista Calidad Asistencial. 1995. p.346-349.

[10] Hernández I, Aibar C, Aranaz JM, et al. "Planificación y programación en atención a la salud" En:Piédrola G. et al, editores. Medicina Preventiva y Salud Pública. Madrid: Elsevier, 2008. p.1191-1198.

[11] Diego-Martin F, Ortega-Mier M, Garcia-Sanchez A, et al."Strategic planning problem of staff to cover any claim temporary work load of variable". DYNA. 2015.Vol.90. p.1-8. DOI: http://dx.doi.org/10.6036/7248.

[12] Limpert E, Stahel W, Abbt M. Log-normal Distributions across the Sciences: Keys and Clues. BioScience. 2001. Vol.51-5. p.341-352. DOI http://dx.doi.org/10.1641/0006-3568(2001)051[0341:LNDATS]2.0.CO;2.

[13] Atienza G, Arruti E, Ceinos E, et al. "Estudio de la demanda asistencial en un servicio de urgencias hospitalario II". Emergencias. 1993. Vol.51. p.11-14.

[14] García J, Claveria A. "Tiempo de estancia en un Servicio de Urgencias Hospitalario". Emergencias. 1989. Vol.1. p.17-21.

[15] Von Neumann J, Ulam S. "Monte Carlo Method". National Bureau of Standards symposium. Applied Mathematics Series. National Bureau of Standards. Washington D.C.; 1951, 12, 36.

[16] Pineault R. La Planificación Sanitaria. Barcelona: Editorial Masson, 1989. 400 p.ISBN: 978-84-311-0468-9.

[17] Almehdawe E, Jewkes B, He QM. A Markovian queueing model for ambulance offload delays. European Journal of Operational Research. 2013. Vol.226. p.602-614. DOl:http://dx.doi.org/10.1016/j.ejor.2012.11.030.

[18] Naoum-Sawaya J, Elhedhli S. A stochastic optimization model for real-time ambulance redeployment. Computers \& Operations Research. 2013. Vol.40. p.1972-1978. DOl:http://dx.doi.org/10.1016/j.cor.2013.02.006. 